\title{
Cofactor engineering through heterologous expression of an NADH oxidase and its impact on metabolic flux redistribution in Klebsiella pneumoniae
}

\author{
Xiao-Jun Ji ${ }^{\dagger}$, Zhi-Fang $\mathrm{Xia}^{\dagger}{ }^{\dagger}$ Ning-Hua Fu, Zhi-Kui Nie, Meng-Qiu Shen, Qian-Qian Tian and He Huang ${ }^{*}$
}

\begin{abstract}
Background: Acetoin is an important bio-based platform chemical. However, it is usually existed as a minor byproduct of 2,3-butanediol fermentation in bacteria.

Results: The present study reports introducing an exogenous $\mathrm{NAD}^{+}$regeneration sysytem into a 2,3-butanediol producing strain Klebsiella pneumoniae to increse the accumulation of acetoin. Batch fermentation suggested that heterologous expression of the NADH oxidase in K. pneumoniae resulted in large decreases in the intracellular $\mathrm{NADH}$ concentration ( 1.4 fold) and NADH/NAD ${ }^{+}$ratio $(2.0$ fold). Metabolic flux analysis revealed that fluxes to acetoin and acetic acid were enhanced, whereas, production of lactic acid and ethanol were decreased, with the accumualation of 2,3-butanediol nearly unaltered. By fed-batch culture of the recombinant, the highest reported acetoin production level $(25.9 \mathrm{~g} / \mathrm{L})$ by Klebsiella species was obtained.

Conclusions: The present study indicates that microbial production of acetoin could be improved by decreasing the intracellular NADH/NAD ${ }^{+}$ratio in K. pneumoniae. It demonstrated that the cofactor engineering method, which is by manipulating the level of intracellular cofactors to redirect cellular metabolism, could be employed to achieve a high efficiency of producing the $\mathrm{NAD}^{+}$-dependent microbial metabolite.
\end{abstract}

Keywords: Acetoin, 2,3-Butanediol, Cofactor engineering, Klebsiella pneumoniae, NADH oxidase

\section{Background}

Metabolic engineering has been widely applied in modifying metabolic pathways to improve the properties of microbial strains, including manipulation of enzyme levels through the amplification, disruption, or addition of a metabolic pathway [1]. These approaches have been proven to be powerful in developing microbial strains for the commercial production of organic acids, amino acids, biofuels, and pharmaceuticals [2]. Nevertheless, overexpression, deletion, or introduction of heterologous genes in target metabolic pathways do not always result in the desired phenotype [3]. In recent years, cofactor engineering,

\footnotetext{
*Correspondence: biotech@njut.edu.cn

${ }^{\dagger}$ Equal contributors

State Key Laboratory of Materials-Oriented Chemical Engineering, College of Biotechnology and Pharmaceutical Engineering, Nanjing University of Technology, No. 5 Xinmofan Road, Nanjing 210009, People's Republic of China
}

\section{Biomed Central}

(c) $2013 \mathrm{Ji}$ et al.; licensee BioMed Central Ltd. This is an Open Access article distributed under the terms of the Creative Commons Attribution License (http://creativecommons.org/licenses/by/2.0), which permits unrestricted use, distribution, and reproduction in any medium, provided the original work is properly cited. which is considered as a new branch of metabolic engneering, has attracted increasing attention. Manipulations of the cofactor form and level have become a useful tool for metabolic engineering to redistribute/enhance carbon flux in metabolic networks [4]. Nicotinamide adenine dinucleotides $\left(\mathrm{NADH}\right.$ and $\mathrm{NAD}^{+}$), as one pair of key cofactors play an important role in over 300 biochemical reactions involving oxidation and reduction $[2,5]$. Therefore, this cofactor pair $\left(\mathrm{NADH}\right.$ and $\mathrm{NAD}^{+}$) has a critical effect on maintaining the intracellular redox balance, which is a basic condition for microorganism to metabolize and grow [6]. Regulation of the $\mathrm{NADH} / \mathrm{NAD}^{+}$ratio can be achieved by either weakening the metabolic pathways competing for NADH or $\mathrm{NAD}^{+}$[7-9], or introducing an $\mathrm{NADH}$ or $\mathrm{NAD}^{+}$regeneration system. Intracellular concentrations of $\mathrm{NADH}$ and $\mathrm{NAD}^{+}$can be changed by expressing an $\mathrm{NAD}^{+}$-dependent formate dehydrogenase (EC 1.2.1.2; $\mathrm{FDH}$ ), an $\mathrm{NADH}$ 
oxidase (EC 1.6.99.3; NOX), or a nicotinic acid phosphoribosyl transferase (EC 2.4.2.11; NAPRTase). Increase of intracellular $\mathrm{NADH}$ availability by overexpressing $\mathrm{FDH}$ in bacteria provoked a significant metabolic redistribution $[10,11]$. Heterologous expression of NOX was conducted in Lactococcus latis, resulting in a low $\mathrm{NADH} / \mathrm{NAD}^{+}$ratio and the shift from homolactic fermentation to mixed-acid fermentation under aerobic conditions [4]. Overexpressing the gene of $p n c \mathrm{~B}$ encoding NAPRTase in $E$. coli, was observed to increase the total $\mathrm{NAD}^{+}$level and decrease the $\mathrm{NADH} / \mathrm{NAD}^{+}$ratio [12]. These studies showed that the expression of the $\mathrm{NADH}$ or $\mathrm{NAD}^{+}$-related enzymes could lead to a dramatically altered $\mathrm{NADH} / \mathrm{NAD}^{+}$ratio and a significantly changed spectrum of metabolic products [11].

Cofactor engineering has been successfully applied for the prodcution of many bio-based chemicals, such as 1,3-propanediol [9,11], pyruvic acid [13], and succinic acid [14]. In the present work, this novel and powerful technology will be applied to the production of another important bio-based chemical, acetoin. Acetoin is defined as one of the high value-added platform compounds and selected by the U. S. Department of Energy as one of the potential top 30 chemical building blocks from sugars [15]. A number of microorganisms are able to accumulate acetoin, including the genera Klebsiella, Paenibacillus, Bacillus, Serratia, etc. [16,17]. However, these strains are widely known as good producers of 2,3-butanediol (2,3-BD) which is another important bio-based platform chemical [18]. Acetoin is only generated as a minor by-product. Although some Bacillus strains have been used for acetoin production [19-21], the long fermentation period generally needed in fermentative acetoin using Bacillus strains still hinder its large-scale production.

In bacteria, acetoin and 2,3-BD are produced by the mixed acid-2,3-BD fermentation pathway. In the presence of $\mathrm{NAD}^{+}, 2,3-\mathrm{BD}$ dehydrogenase (EC 1.1.1.76; $\mathrm{BDH})$, which is a NADH-dependent dehydrogenase, can catalyze 2,3-BD to acetoin [22] (Additional file 1: Figure S1). Due to the reversible transformation between acetoin and 2,3-BD coupled with the NADH/ $\mathrm{NAD}^{+}$conversion, the synthesis of these two products have been considered involving in regulation of the $\mathrm{NADH} / \mathrm{NAD}^{+}$ratio in bacteria [23]. That is to say, these two products exist in the same branch of the metabolic pathway linked with $\mathrm{NADH}$ and $\mathrm{NAD}^{+}$ transformation. Considering that Klebsiella species are the most powerful 2,3-BD producers, they could be engineered to obtain high acetoin producing ability. In this work, an $\mathrm{NAD}^{+}$regeneration system (nox-2 gene from Streptococcus pneumoniae, encoding NOX) was introduced into a 2,3-BD producing strain Klebsilla pneumoniae to manipulate the intracellular $\mathrm{NAD}^{+}$level and $\mathrm{NADH} / \mathrm{NAD}^{+}$ratio for acetoin overproduction. The consequent effect on the distribution of metabolites in
K. pneumoniae, especially, on the production of compounds that require $\mathrm{NADH}$ or $\mathrm{NAD}^{+}$for their synthesis, including acetoin, 2,3-BD, and other by-products, such as ethanol, lactic acid, and acetic acid, was investigated.

\section{Results}

\section{Expression of the $S$. pneumoniae nox-2 gene in} K. pneumoniae and its effect on cell growth

To investigate the effect of $\mathrm{NAD}^{+}$regeneration on acetoin production, the nox-2 gene from S. pneumoniae encoding the water-forming NOX was cloned and overexpressed in a 2,3-BD producing strain $K$. pneumoniae. As indicated in the section of 'Materials and methods', the confirmed recombinant XZF-308 was obtained. The NOX activities of the recombinant and the parent strain were determined. As shown in Figure 1, the highest specific activity of $\mathrm{NOX}(1.57 \mathrm{U} / \mathrm{mg}$ protein) was found in the recombinant strain when cells entered exponential growth phase. While NOX activity in the parent strain was extremely low during the entire fermentation period, revealing that NOX was positively produced in the recombinant strain (Additional file 2: Figure S2).

To investigate the effect of NOX overexpression on cell growth, the recombinant strain and the parent strain were cultured at the same conditions. As shown in Figure 2, the recombinant strain grew to a lower $\mathrm{OD}_{600}$ as compared to the parent strain, indicating that the cell growth of K. pneumoniae was inhibited due to the heterologous expression of NOX. At the first $8 \mathrm{~h}$, the cell growth rate of the recombinant strain $(0.18 \mathrm{~g} /(\mathrm{L} \cdot \mathrm{h}))$ reached only around half of that the parent strain achieved $(0.38 \mathrm{~g} /(\mathrm{L} \cdot \mathrm{h}))$. The growth rates for both strains were then increased $(8-11 \mathrm{~h})$ but the growth rate of the recombinant strain $(0.63 \mathrm{~g} /(\mathrm{L} \cdot \mathrm{h}))$ was still lower compared to the parent strain $(0.76 \mathrm{~g} /(\mathrm{L} \cdot \mathrm{h}))$. At the stage of $11-14 \mathrm{~h}$,

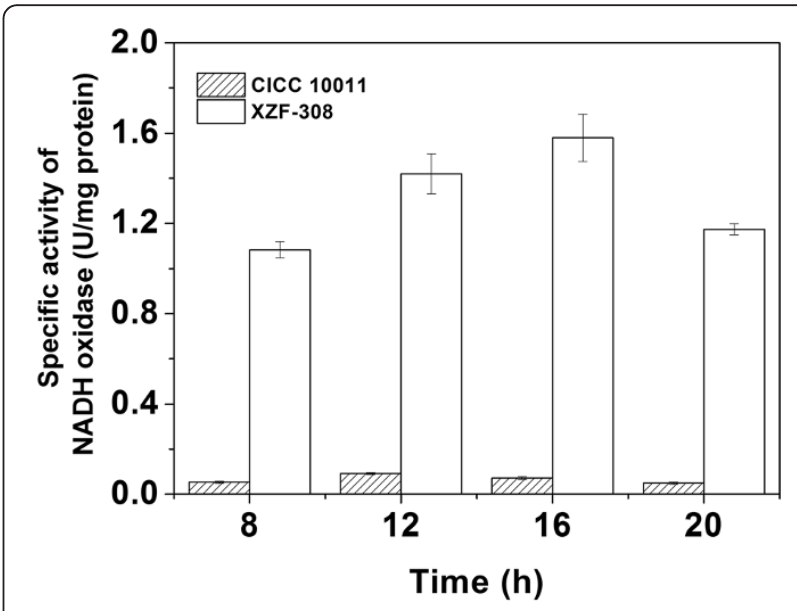

Figure 1 Time courses of NADH oxidase activity of the parent and the recombinant Klebsiella pneumoniae. CICC 10011: the parent strain; XZF-308: the recombinant strain. 


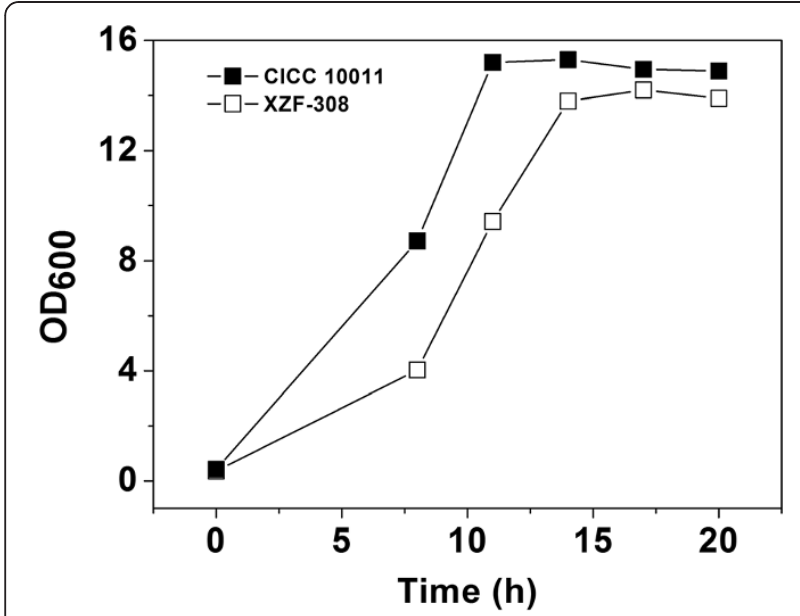

Figure 2 Growth curves of the parent and the recombinant Klebsiella pneumoniae. CICC 10011: the parent strain; XZF-308: the recombinant strain.

although the recombinant strain grew faster than the parent strain, its biomass accumulation was still lower than the parent strain, and this trend lasted to the end.

\section{Effect of the heterologous NOX expression on} intracellular concentrations of NADH and NAD ${ }^{+}$

Heterologous expression of NOX in K. pneumoniae was expected to decrease the total intracellular $\mathrm{NADH}$ pool and thus strengthen the flux of $\mathrm{NAD}^{+}$-dependent pathways. As shown in Figure 3A and B, for both the parent and recombinant strains, the intracellular contents of $\mathrm{NADH}$ and $\mathrm{NAD}^{+}$were constantly changing with the fermentation time. During the growth stage $(0-14 \mathrm{~h})$, the $\mathrm{NADH}$ concentration in the parent strain was increasing firstly and then decreasing, which was different from the declining trend in the recombinant strain. While the NAD ${ }^{+}$levels in both strains were increasing continuously in the whole stage. However, when the cells entered into the non-growth stage (after $14 \mathrm{~h}$ ), the NADH concentration in both strains was increasing and $\mathrm{NAD}^{+}$concentration was decreasing. Furthermore, heterologous expression of NOX in K. pneumoniae led to a lower level of NADH pool and a higher level of $\mathrm{NAD}^{+}$pool when compared to the parent strain. The difference of the $\mathrm{NADH} / \mathrm{NAD}^{+}$ratio between the parent and the recombinant strain was also observed (Figure $3 \mathrm{C}$ ). In fed-batch fermentation, this difference was still existed (Additional file 3: Figure S3). This indicated that the redox status in $K$. pneumoniae was disturbed, which explained why cell growth of the recombinant was negatively affected (Figure 2).

\section{Introduction of $\mathrm{NAD}^{+}$regeneration system leads to} metabolic flux redistribution in $K$. pneumoniae Metabolite profiles of the parent and the recombinant strains were monitored in parallel batch cultures.

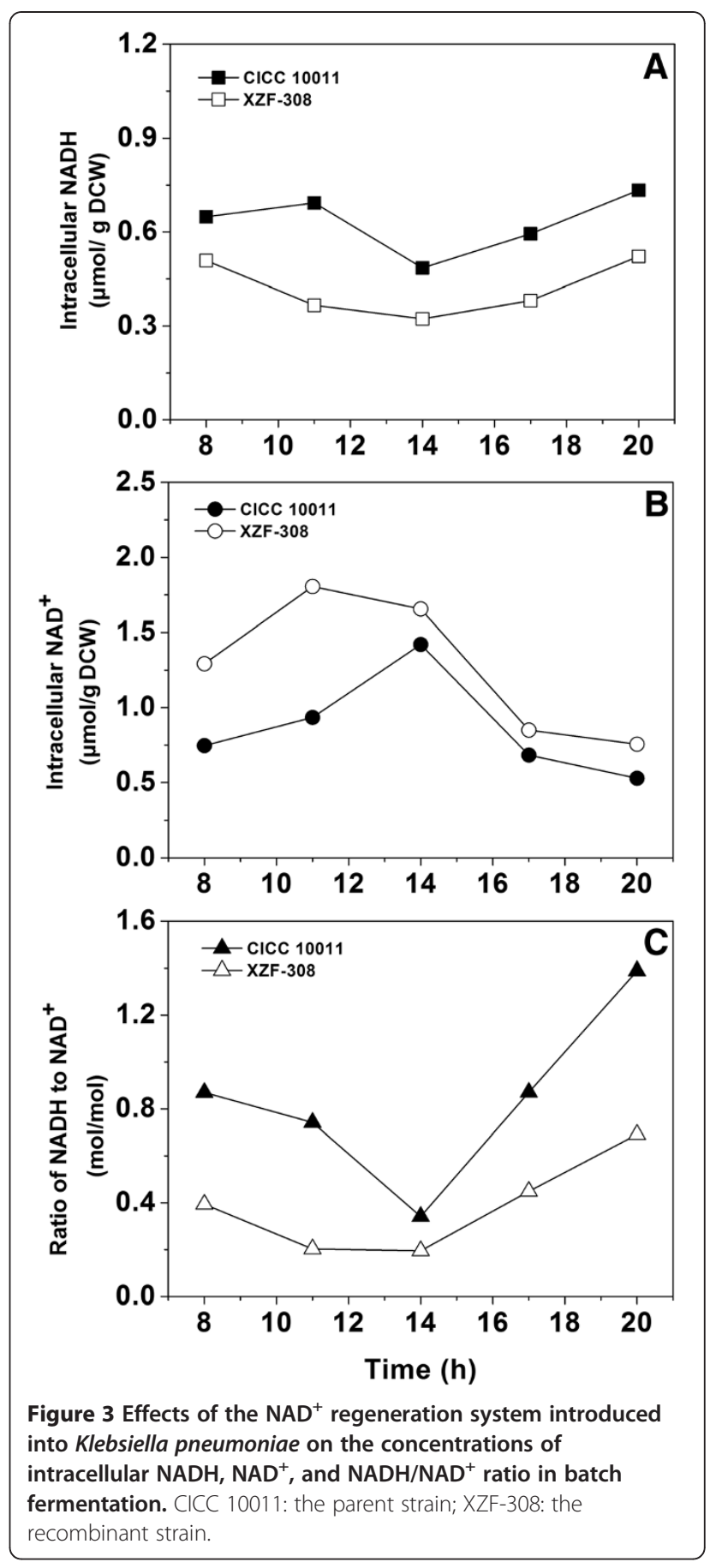

As described in the first part of 'Results' section, the recombinant strain positively expressed NOX, which helped regenerate the intracellular $\mathrm{NAD}^{+}$from NADH. Therefore, the carbon fluxes of the NADH-dependent pathways were expected to be triggered to decrease in the recombinant strain. In the mixed acid-2,3-BD fermentation pathway of $K$. pneumoniae (major products: acetoin and 2,3-BD), the by-products included acetic acid, lactic acid, and ethanol, among which lactic 
acid and ethanol formation competed with 2,3-BD for $\mathrm{NADH}[22]$.

To investigate the metabolic changes of $K$. pneumoniae in response to the heterologous expression of NOX, the concentrations of major metabolites of the recombinant and the parent strains were determined (Figure 4). The recombinant strain produced more acetoin and acetic acid, less ethanol and lactic acid compared to the parent strain probably due to the result of decreased NADH availability. Molar yields of 2,3-BD, lactic acid, and ethanol were $0.5 \%, 12.2 \%$, and $12.9 \%$ lower than those of the parent strain, respectively, while molar yields of acetoin and acetic acid were 189.29\% and 9.5\% higher (Table 1). This suggested that the cellular carbon fluxes were redistributed.

Due to the alteration of intracellular NADH and NAD ${ }^{+}$ pools, the accumulations of acetoin, 2,3-BD, acetic acid, lactic acid, and ethanol in the fermentation broth of the recombinant strain were different from the parent strain. During the fermentation process, acetoin were produced at higher rates by the recombinant strain (Figure 4B), and the final yields of acetoin and acetic acid were also higher compared to the parent strain. However, the final yield of 2,3-BD was similar for both strains, while ethanol and lactic acid production of the parent strain was less. This suggested that the NADH-dependent pathways, including the pathways from pyruvic acid to ethanol, lactic acid, and 2,3-BD, reacted differently in response to the introduction of $\mathrm{NAD}^{+}$regeneration system.

\section{Production of acetoin by fed-batch culture of the recombinant $K$. pneumoniae}

As shown in Figure 4B, it was observed that the acetoin titer could not further increase when the glucose was exhausted. Therefore, a fed-batch culture by feeding glucose solution was carried out (Additional file 4: Figure S4). After $80 \mathrm{~h}$, the final acetoin titer reached $25.8 \mathrm{~g} / \mathrm{L}$ (Figure 5B). While the time courses of the other metabolites had the same tendency with the batch culture (Figure 5A, D, E, F). In the previous studies, K. pneumoniae

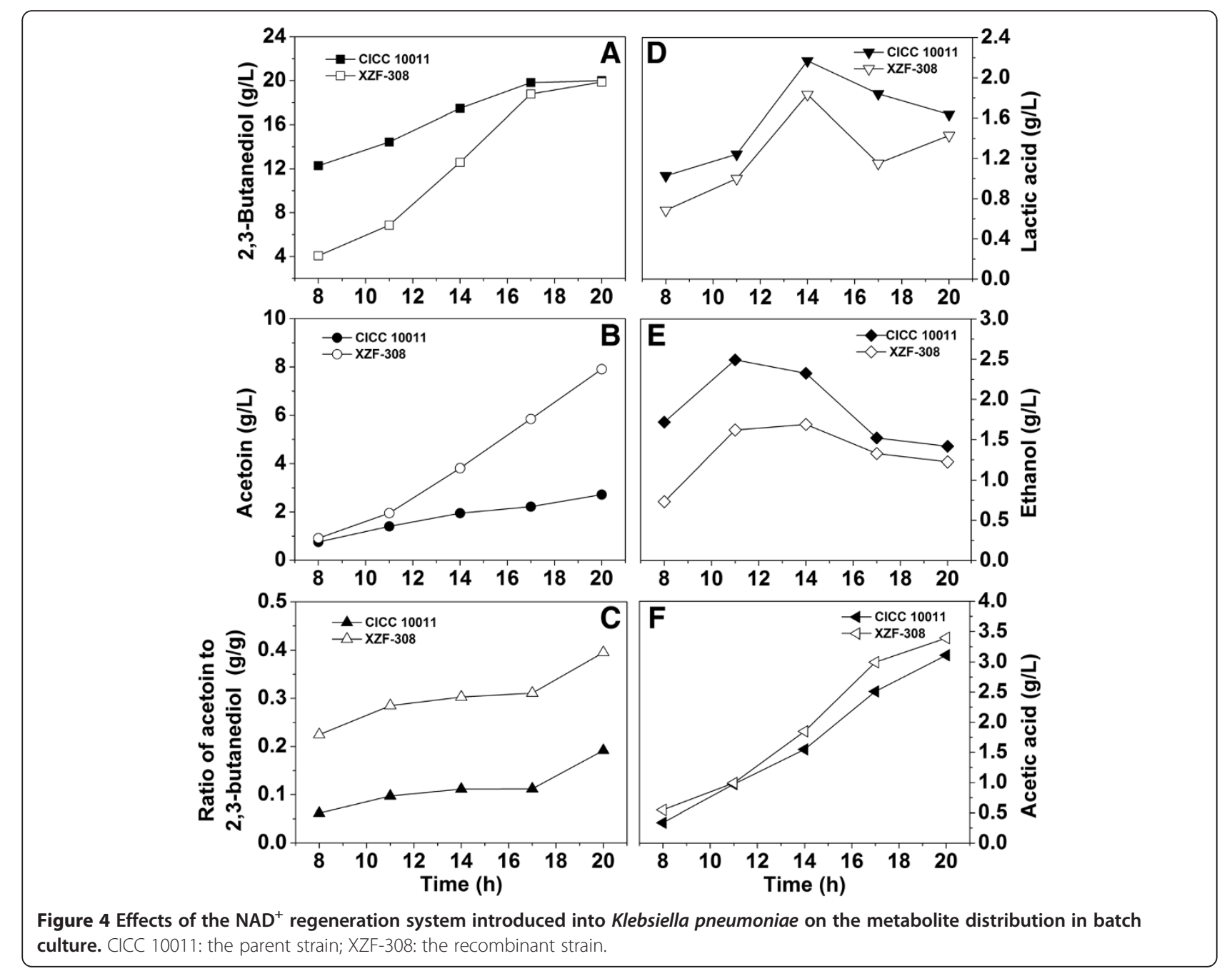


Table 1 Effect of the $\mathrm{NAD}^{+}$regeneration system introduction on the metabolic flux distribution in Klebsiella pneumoniae (unit: $\mathrm{mol} / \mathrm{mol}$ glucose)

\begin{tabular}{lccc}
\hline Metabolites $^{\mathbf{a}}$ & $\boldsymbol{K}_{\text {. pneumoniae CICC10011 }}$ & $\boldsymbol{K}_{\text {. pneumoniae }}$ XZF-308 & Fluxes redistributed by introducing the NAD ${ }^{+}$regeneration system \\
\hline Acetoin & 0.056 & 0.162 & $189.29 \%$ \\
2,3-Butanediol & 0.400 & 0.398 & $-0.5 \%^{\mathrm{b}}$ \\
Acetic acid & 0.093 & 0.102 & $9.5 \%$ \\
Lactic acid & 0.033 & 0.029 & $-12.2 \%^{\mathrm{b}}$ \\
Ethanol & 0.055 & 0.048 & $-12.9 \%^{\mathrm{b}}$ \\
\hline
\end{tabular}

${ }^{a}$ Acetoin is an $\mathrm{NAD}^{+}$dependent metabolite, 2,3-butanediol, lactic acid, and ethanol are NADH dependent metabolites, while acetic acid is not dependent of $\mathrm{NADH}$ or $\mathrm{NAD}^{+}$.

${ }^{\mathrm{b}}$ Negative standard means a decrease in glucose flux to 2,3-butanediol, lactic acid, and ethanol.

and another Klebsiella species-Klebsiella oxytoca was used for 2,3-BD production, acetoin which acted as a kind of coupled by-product was collected accompanying with 2,3$\mathrm{BD}$ at the end of fermentation. However, its production titer was generally lower than $15 \mathrm{~g} / \mathrm{L}$. Here, the engineered K. pneumoniae could accumulate more than $20 \mathrm{~g} / \mathrm{L}$ acetoin, which is the highest report of acetoin yield by Klebsiella species (Table 2).

\section{Discussion}

In the present study, the heterologous NOX was successfully expressed in $K$. pneumoniae and the effect of
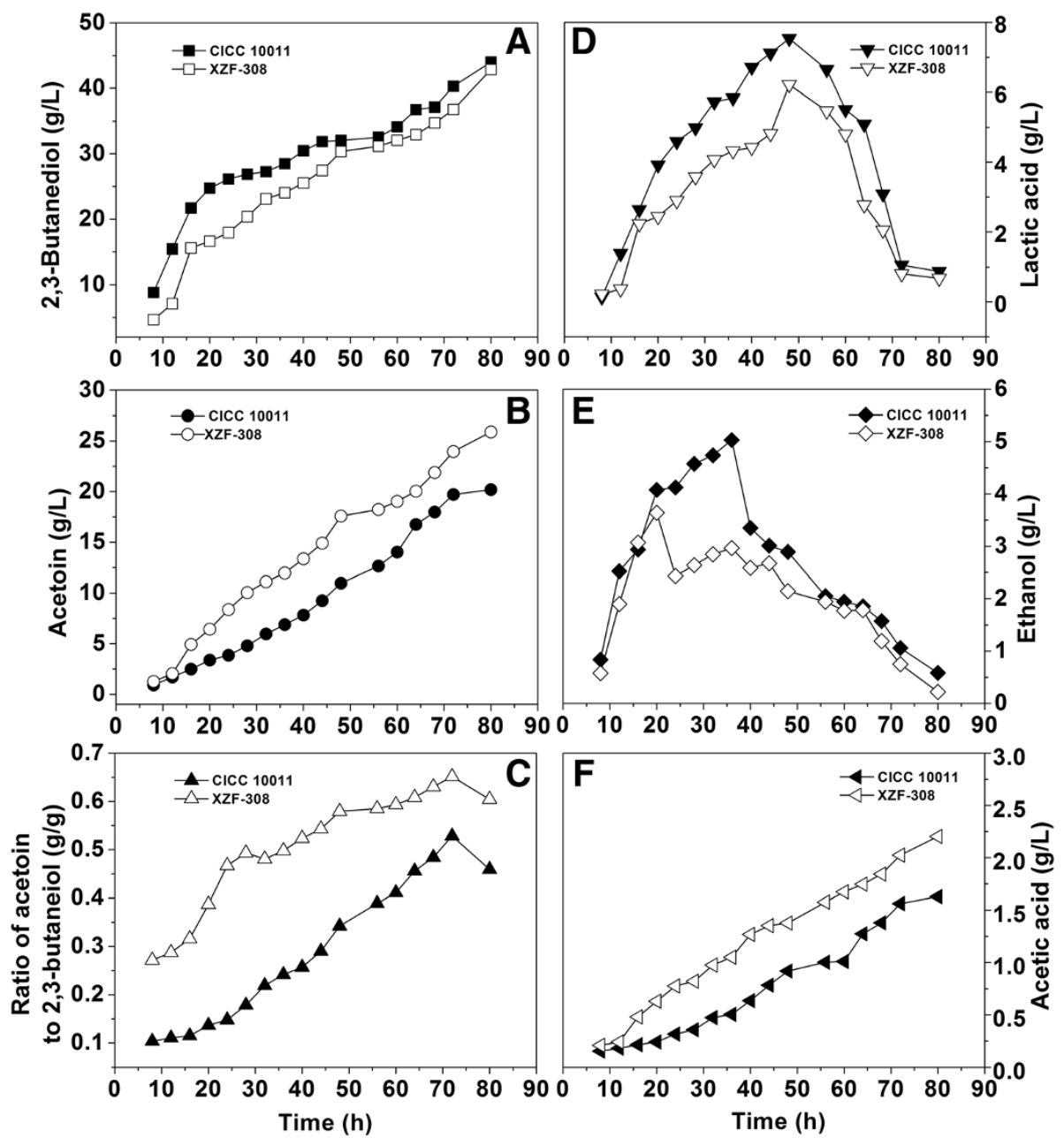

Figure 5 Effects of the $\mathrm{NAD}^{+}$regeneration system introduced into Klebsiella pneumoniae on the metabolite distribution in fed-batch culture. CICC 10011: the parent strain; XZF-308: the recombinant strain. 
Table 2 Microbial production of acetoin and 2,3-butanediol using Klebsiella species

\begin{tabular}{lccc}
\hline Strains & \multicolumn{2}{c}{ Products (g/L) } & References \\
\cline { 2 - 3 } & Acetoin & 2,3-Butanediol & \\
\hline Klebsiella oxytoca & 2.3 & 118.0 & {$[24]$} \\
Klebsiella oxytoca & 1.9 & 95.5 & {$[25]$} \\
Klebsiella oxytoca & 1.4 & 130.0 & {$[8]$} \\
Klebsiella oxytoca & 15.4 & 86.2 & {$[26]$} \\
Klebsiella oxytoca & 6.4 & 85.5 & {$[27]$} \\
Klebsiella pneumoniae & 13.1 & 67.4 & {$[28]$} \\
Klebsiella pneumoniae & 10.0 & 150.0 & {$[29]$} \\
Klebsiella pneumoniae & 13.1 & 92.4 & {$[30]$} \\
Klebsiella pneumoniae & 7.6 & 84.0 & {$[31]$} \\
Klebsiella pneumoniae & 3.6 & 78.9 & {$[32]$} \\
Klebsiella pneumoniae & 25.9 & 42.8 & This work \\
\hline
\end{tabular}

introducing $\mathrm{NAD}^{+}$regeneration system on glucose metabolism was investigated. After conducting the cofactor engineering manipulations, the intracellular redox status (showed by the NADH/NAD ${ }^{+}$ratio) was disturbed. The result was similar to that of engineered $S$. cerevisiae $[6,33,34]$ and L. lactis [4], in which the NADH/NAD ${ }^{+}$ ratio was greatly influenced by introducing the system of $\mathrm{NAD}^{+}$regeneration. Owing to changed intracellular redox status, the cell growth of the recombinant $K$. pneumoniae was somewhat inhibited.

The heterologous expression of NOX in K. pneumoniae was a useful tool for qualifying the metabolic network and studying the interaction between the NADH level and metabolic fluxes. As indicated in the third part of the
'Results' section, the large decrease of the NADH/NAD' ratio due to the introduction of the $\mathrm{NAD}^{+}$regeneration system triggered a dramatic metabolic flux redistribution, as shown by changed concentrations of acetoin, 2,3-BD, acetic acid, lactic acid, and ethanol. Generally, in the mixed acid-2,3-BD fermentation pathway, the synthesis of 2,3-BD requires a large number of $\mathrm{NADH}$ along with the reduction of acetoin. Besides, the formation of lactic acid and ethanol also needs $\mathrm{NADH}$ and therefore the productivity of these products is related with intracellular NADH and $\mathrm{NAD}^{+}$concentrations. With the decrease of NADH concentration in the recombinant strain, the acetoin production was almost tripled compared to the parent strain in the batch culture (Figure 4B), whereas the 2,3-BD production was nearly the same as the parent strain, which was not anticipated to decrease after the introduction of the $\mathrm{NAD}^{+}$regeneration system. At the same time, in the recombinant, the carbon fluxes to lactic acid and ethanol were decreased due to the fact that the NADH availability was reduced. That is to say, the increase of the acetoin production was at the expense of lactic acid and ethanol production while not 2,3-BD, although accumulation of these metabolites all require NADH.

In fact, in response to the introduction of $\mathrm{NAD}^{+}$regeneration system, the first to be affected were the lactic acid and ethanol metabolic pathways among the three NADH dependent pathways. After introducing the $\mathrm{NAD}^{+}$regeneration system by heterologous expression of NOX, the excess NADH generated by the glycolytic pathway that would be channeled to the respiratory chain to form ATP, was oxidized to $\mathrm{H}_{2} \mathrm{O}$ catalyzed by the NOX. Therefore, the acetic acid pathway was triggered to be

Table 3 Bacterial strains, plasmids, and primers used in this study

\begin{tabular}{|c|c|c|}
\hline Strains, plasmids, or primers & Genotypes, properties, or sequences $^{a}$ & Sources or references \\
\hline \multicolumn{3}{|l|}{ Strains } \\
\hline Klebsiella pneumoniae CICC10011 & $A p^{r}$, parent strain & $\mathrm{CICC}$ \\
\hline K. pneumoniae XZF-308 & $\mathrm{Ap}^{r}, \mathrm{Cm}^{r}$, recombinant strain of CICC10011, harbring pDK7-nox-2 & This work \\
\hline Escherichia coli DH5a & supE44 $\Delta$ lacU169 ( $(80$ lacZ $\triangle$ M15) hsdR17 recA1 endA1 gyrA96 thi-1 relA1 & TaKaRa \\
\hline \multicolumn{3}{|l|}{ Plasmids } \\
\hline pNOX2 & pMosBlue carrying a PCR product encoding NADH oxidase of S. pneumoniae & [38] \\
\hline pUC18 & $A p^{r}, 2686$ bp & TaKaRa \\
\hline pUC18-nox-2 & $\begin{array}{l}\text { Apr', pUC18 derivative carrying a } 1380 \text { bp DNA fragment containing the gene } \\
\text { of nox-2, } 4066 \mathrm{bp}\end{array}$ & This work \\
\hline pDK7 & Expressing vector, $\mathrm{Cm}^{\mathrm{r}}, 4800 \mathrm{bp}$ & [39] \\
\hline pXZF-308 & $\begin{array}{l}\mathrm{Cm}^{\mathrm{r}} \text {, pDK7 derivative carrying a } 1380 \mathrm{bp} \text { DNA fragment containing the gene } \\
\text { of nox-2, } 6180 \mathrm{bp}\end{array}$ & This work \\
\hline \multicolumn{3}{|l|}{ Primers ${ }^{b}$} \\
\hline P1 & 5'-CGGGGTACCTAAGGAGGATATACATATGAGTAAAATCGT-3' (Kpn I) & This work \\
\hline P2 & 5'-CGGCTGCAGTTATTTTTCAGCCGTAAG-3' (Pst I) & This work \\
\hline
\end{tabular}

${ }^{a} A p^{r}$ : ampicillin resistance; $\mathrm{Cm}^{\mathrm{r}}$ : chloramphenicol resistance.

${ }^{\mathrm{b}}$ The underlined letters indicate the restriction sites. 
enhanced as it could alternatively contribute to generate ATP which was essential for cell growth (Figure 4F and $5 F)$. This could also explain that why the non-NADH dependent acetic acid metabolic pathway was affected by introducing the $\mathrm{NAD}^{+}$regeneration system. Consequently, due to the enhancement of acetic acid formation, the flux of the metabolic pathway of 2,3-BD was triggered to be increased, as acetic acid was proven to be able to act as an inducer for the production of the key enzymes involved in 2,3-BD formation [22,23]. Therefore, driven by the introduced $\mathrm{NAD}^{+}$regeneration system, the increased fluxes to 2,3-BD pathway was reflected by the increased acetoin accumulation with the 2,3-BD formation nearly unaffected.

\section{Conclusions}

In conclusion, the introduction of $\mathrm{NAD}^{+}$regeneration system into $K$. pneumoniae is a powerful engineering tool to enhance the metabolic flux to some desired metabolites. Increasing the availability of intracellular $\mathrm{NAD}^{+}$favored the production of more oxidative metabolites, as evidenced by a significant increase in the acetoin production, while decrease in the production of lactic acid and ethanol. The present study showed that the NOX can be well expressed in K. pneumoniae, and heterologous expression of NOX resulted in large decreases in the intracellular NADH concentration and $\mathrm{NADH} / \mathrm{NAD}^{+}$ratio, thus altered the metabolite spectrum of the mixed acid-2,3-BD fermentation pathway. To the best of our knowledge, the result in the present study was a new record on acetoin production by Klebsiella species. Furthermore, the result in present study provides useful information for increasing accumulation of $\mathrm{NAD}^{+}$-dependent microbial metabolite. This is another example showing the advantage of cofactor engineering in terms of manipulating the form and level of intracellular cofactors to redirect cellular metabolism. The idea developed in this paper could be applied to the other similar industrial biotechnological process to achieve high product concentration.

\section{Materials and methods}

\section{Strains and plasmid construction}

The strains, plasmids, and primers used in this study were listed in Table 3. K. pneumoniae CICC10011, which was obtained from China Center of Industrial Culture Collection (CICC), was used as the parent strain for acetoin and 2,3-BD production. E. coli DH5 $\alpha$ was used for plasmid cloning and maintenance. The nox-2 gene from S. pneumoniae encoding a water-forming NADH oxidase was amplified by PCR with Taq polymerase (TaKaRa, Dalian, China) using primers P1 and P2 (primers were based on the sequence of the nox-2 gene under GenBank accession No. AF014458). The amplified fragment was digested with Kpn I and Pst I and inserted into a cloning vector pUC18, resulted in pUC18-nox-2. The ampicillin-resistant transformants were selected by the blue/white method on plates. White clones were picked out and the plasmid pUC18-nox-2 was extracted, from which the nox-2 gene was digested by Kpn I and Pst I and introduced into the Kpn I-Pst I site of an expressing vector pDK7 under the control of the tac promoter (Additional file 5: Figure S5). The yielded recombinant plasmid pXZF-308 was transformed into K. pneumoniae CICC10011 by standard transformation protocol using the method of electroporation [35-37]. The chloramphenicol-resistant transformants were selected, and the insert was confirmed by colony PCR, restrictive digestion, and sequencing. The confirmed clone harboring pXZF-308 was designated as $K$. pneumoniae XZF-308.

\section{Media and growth conditions}

Luria-Bertani (LB) medium was used as the seed culture of E. coli, $K$. pneumoniae and their derivatives. When $K$. pneumoniae was used for electroporation, ethylene diamine tetraacetic acid (EDTA) was added to the LB medium to a final concentration of $0.7 \mathrm{mM}$ [37]. After the electroporation, the seed culture for activating the recombinant $K$. pneumoniae was prepared in the SOC medium [40]. When necessary, ampicillin $(60 \mu \mathrm{g} / \mathrm{mL})$ and/or tetracycline $(12 \mu \mathrm{g} / \mathrm{mL})$ was added to the medium as selection markers.

For seeds preparation, K. pneumoniae strains were cultured in a $250-\mathrm{mL}$ Erlenmeyer flask containing $50 \mathrm{~mL}$ fresh medium inoculated with a full loop of K. pneumoniae from fresh slant tube. The seeds were cultivated at $37^{\circ} \mathrm{C}$ and $200 \mathrm{rpm}$ on a rotary shaker for $24 \mathrm{~h}$ [26]. Seed culture $(5 \%, v / v)$ was then inoculated into the fermentation medium and batch and fed-batch fermentation were carried out in a 3-L stirred fermenter (BioFlo 100; New Brunswick Scientific Co., NJ, USA) with a an initial broth volume of $2 \mathrm{~L}$. The fermentation medium for K. pneumoniae was composed of $(\mathrm{g} / \mathrm{L})$ : glucose, 100; $\mathrm{K}_{2} \mathrm{HPO}_{4}, 13.7 ; \mathrm{KH}_{2} \mathrm{PO}_{4}, 2.0 ;\left(\mathrm{NH}_{4}\right)_{2} \mathrm{HPO}_{4}, 3.3 ;\left(\mathrm{NH}_{4}\right)$ ${ }_{2} \mathrm{SO}_{4}, \quad 6.6 ; \quad \mathrm{MgSO}_{4} \cdot 7 \mathrm{H}_{2} \mathrm{O}, 0.25 ; \quad \mathrm{FeSO}_{4} \cdot 7 \mathrm{H}_{2} \mathrm{O}, 0.05$; $\mathrm{ZnSO}_{4} \cdot 7 \mathrm{H}_{2} \mathrm{O}, 0.001 ; \mathrm{MnSO}_{4} \cdot \mathrm{H}_{2} \mathrm{O}, 0.001 ; \mathrm{CaCl}_{2} \cdot 2 \mathrm{H}_{2} \mathrm{O}$, 0.01; EDTA, 0.05 [8]. The fermentation was performed at $37^{\circ} \mathrm{C}$ with the aeration rate of $1.0 \mathrm{vvm}$ and agitation speed of $400 \mathrm{rpm}$, respectively. When the $\mathrm{pH}$ decreased to 6.5 , it was controlled at 6.5 automatically by adding $3 \mathrm{M} \mathrm{NaOH}$. Fed-batch fermentation was conducted by feeding $400 \mathrm{~g} / \mathrm{L}$ glucose when the residual glucose in the fermentation broth was below $10 \mathrm{~g} / \mathrm{L}$.

\section{Preparation of cell extracts and NOX activity assay}

The recombinant strain $K$. pneumoniae XZF-308 was grown in 500-mL Erlenmeyer flasks with $100 \mathrm{~mL}$ working 
volume and also cultured in 3-L fermentor with $2 \mathrm{~L}$ working volume. Isopropyl $\beta$-d-thiogalactoside (IPTG) was added into the culture to a final concentration of $1 \mathrm{mM}$ to induce the expression of nox-2 gene when the strains grew to an $\mathrm{OD}_{600}$ of $0.6-0.8$. After induction at $32^{\circ} \mathrm{C}$ for $10 \mathrm{~h}$, cell pellets were washed with $35 \mathrm{mM}$ potassium phosphate ( $\mathrm{pH}$ 8.0) buffer twice, and resuspended in the same buffer. Cells were then disrupted by sonication at $4^{\circ} \mathrm{C}$ for $10 \mathrm{~min}$. Cell debris was removed by centrifugation at $12,000 \mathrm{rpm}$ and $4^{\circ} \mathrm{C}$ for $20 \mathrm{~min}$. The supernatant was used for NOX activity assay. According to the previous studies [41], NOX activity was assayed spectrophotometrically at $25^{\circ} \mathrm{C}$ in a total volume of $1 \mathrm{~mL}$ containing $50 \mathrm{mM}$ potassium phosphate (pH 7.0), 0.3 mM EDTA, $10 \mu \mathrm{M}$ FAD, and $0.29 \mathrm{mM}$ NADH. The reaction was initiated by adding a suitable amount of cell extracts to the reaction mixture and the decrease in absorbance at $340 \mathrm{~nm}$ was determined to calculate NADH concentration. A unit of NOX activity was defined as the amount that catalyzed the oxidation of $1 \mu \mathrm{mol}$ of $\mathrm{NADH}$ to $\mathrm{NAD}^{+}$per minute at $25^{\circ} \mathrm{C}$. Total protein concentration in cell extracts was determined by the method described by Bradford with bovine serum albumin as standard protein [42].

\section{Analytical methods}

Biomass, which was shown as dry cell weight (DCW, g/L), was determined by measuring the turbidity of the culture at $600 \mathrm{~nm}$ using a UV visible spectroscopy system (Lambda25, Perkin-Elmer, USA). One unit of optical density was determined to be equivalent to $0.35 \mathrm{~g}$ DCW per liter. The concentration of acetoin, 2,3-BD, and other by-products (lactic acid, acetic acid, and ethanol) were measured by a high-performance liquid chromatography (Summit P680 HPLC, Dionex, USA; Shodex RI-101 Refractive Index Detector, Showa Denko, Japan; Aminex HPX-87H Ion Exclusion Column $300 \mathrm{~mm} \times 7.8 \mathrm{~mm}$, Bio-Rad, USA) under the following conditions: sample volume $10 \mu \mathrm{L}$; mobile phase $0.005 \mathrm{M} \mathrm{H}_{2} \mathrm{SO}_{4}$; flow rate $0.6 \mathrm{~mL} / \mathrm{min}$; column temperature $60^{\circ} \mathrm{C}$.

The intracellular $\mathrm{NADH}$ and $\mathrm{NAD}^{+}$concentrations were measured by procedures presented by San et al. [1]. One milliliter culture broth was sampled from the reactor and quickly pipetted into a tube. The samples were then centrifuged at $12,000 \mathrm{rpm}$ for $5 \mathrm{~min}$. The supernatant was removed and $300 \mu \mathrm{L} 0.2 \mathrm{~mol} / \mathrm{L} \mathrm{NaOH}$ for $\mathrm{NADH}$ or $300 \mu \mathrm{L} 0.2 \mathrm{~mol} / \mathrm{L} \mathrm{HCl}$ for $\mathrm{NAD}^{+}$was added to the pellets to resuspend them. The $\mathrm{NaOH}$ extraction destroyed the oxidized form, and the $\mathrm{HCl}$ extraction destroyed the reduced form of pyridine nucleotides [43]. The samples were placed in a water bath at $50^{\circ} \mathrm{C}$ for $10 \mathrm{~min}$ and then transferred to an ice bath for cooling down to $0^{\circ} \mathrm{C}$. After neutralization by adding $300 \mu \mathrm{L} 0.1 \mathrm{~mol} / \mathrm{L} \mathrm{HCl}$ for $\mathrm{NADH}$ extraction or $300 \mu \mathrm{L} 0.1 \mathrm{~mol} / \mathrm{L} \mathrm{NaOH}$ for $\mathrm{NAD}^{+}$extraction, the samples were centrifuged at $12,000 \mathrm{rpm}, 4^{\circ} \mathrm{C}$ for $10 \mathrm{~min}$. The cell debris was removed, and the supernatant was transferred to a new tube and stored at $-20^{\circ} \mathrm{C}$. The intracellular $\mathrm{NADH}$ or $\mathrm{NAD}^{+}$concentration was determined by the enzymatic cycling assay method [44]. The mixture of cycling asssay consisted of equal volumes of buffer (Bicine buffer, $1.0 \mathrm{~mol} / \mathrm{L}, \mathrm{pH} 8.0$ ), absolute ethanol, EDTA (40 mmol/L, pH 8.0), thiazolyl-blue (MTT, $4.2 \mathrm{mmol} / \mathrm{L}$ ), and twice the volume of phenanzinium ethylsulfate (PES, $16.6 \mathrm{mmol} / \mathrm{L}$ ), was incubated at $30^{\circ} \mathrm{C}$ for $10 \mathrm{~min}$. The reaction mixture contained $50 \mu \mathrm{L}$ neutralized cell extraction, $0.3 \mathrm{~mL}$ purified water, $0.6 \mathrm{~mL}$ mixture referred above, and $50 \mu \mathrm{L}$ alcohol dehydrogenase (EC 1.1.1.1; $500 \mathrm{U} / \mathrm{mL})$. The absorbance at $570 \mathrm{~nm}$ was checked for $10 \mathrm{~min}$. The concentrations of $\mathrm{NADH}$ and $\mathrm{NAD}^{+}$in the supernatant were determined, and the content was calculated by a linear fit equation. This equation was obtained from the slope of the linear region of the absorbance versus time plot, correlated with the concentrations of NADH and NAD ${ }^{+}$.

\section{Additional files}

Additional file 1: Metabolic pathways of acetoin and 2,3-butanediol in the bacteria.

Additional file 2: Validation of the expression of NADH oxidase in Klebsiella pneumoniae through SDS-PAGE.

Additional file 3: Strategy for feeding glucose during the fed-batch fermentation process.

Additional file 4: The intracellular oxidation-reduction level was affected by expressing heterologous NADH oxidase in Klebsiella pneumoniae in the fed-batch fermentation.

Additional file 5: Strategies for constructing the recombinant plasmids.

Competing interests

The authors declare that they have no competing interests.

Authors' contributions

XJJ and HH designed experiments. ZFX, ZKN, NHF, and XJJ performed experiments. MQS and QQT contributed reagents and materials. ZFX and ZKN analyzed data. ZFX, XJJ, and HH wrote the manuscript. All authors have read and approved the final manuscript.

\section{Acknowledgements}

This work was financially supported by the National Natural Science Foundation of China (No. 21006049), the National Key Basic Research and Development Program of China (No. 2011CBA00807), the National Science Foundation for Distinguished Young Scholars of China (No. 21225626), the National High Technology Research and Development Program of China (No. 2011AA02A207), the Key Project of Jiangsu Natural Science Foundation (No. BK2011031), the Program for Changjiang Scholars and Innovative Research Team in University (No. IRT1066), and the Priority Academic Program Development of Jiangsu Higher Education Institutions. The authors thank Prof. Mike J. Merrick (John Innes Centre, UK) and Prof. Liming Liu (Jiangnan University, China) for providing the pDK7 and pNOX2 plasmids.

Received: 19 December 2012 Accepted: 23 January 2013

Published: 25 January 2013 


\section{References}

1. San KY, Bennett GN, Berríos-Rivera SJ, Vadali RV, Yang YT, Horton E, Rudolph FB, Sariyar B, Blackwood K: Metabolic engineering through cofactor manipulation and its effects on metabolic flux redistribution in Escherichia coli. Metab Eng 2002, 4:182-192.

2. Liu LM, Chen J: Cofactor engineering enhances the physiological function of an industrial strain. In Progress in Molecular and Environmental Bioengineering-From Analysis and Modeling to Technology Applications. Edited by Carpi A. Rijeka: InTech Open Access Publisher; 2011:427-444.

3. Jang YS, Park JM, Choi S, Choi YJ, Seung DY, Cho JH, Lee SY: Engineering of microorganisms for the production of biofuels and perspectives based on systems metabolic engineering approaches. Biotechnol Adv 2011, 30:989-1000.

4. de Felipe FL, Kleerebezem M, de Vos WM, Hugenholtz J: Cofactor engineering: a novel approach to metabolic engineering in Lactococcus lactis by controlled expression of NADH oxidase. J Bacterio/ 1998, 180:3804-3808.

5. Foster JW, Park YK, Penfound T, Fenger T, Spector MP: Regulation of NAD metabolism in Salmonella typhimurium: molecular sequence analysis of the bifunctional nadR regulator and the nadA-pnuC operon. J Bacteriol 1990, 172:4187-4196.

6. Heux S, Cachon R, Dequin S: Cofactor engineering in Saccharomyces cerevisiae: expression of a $\mathrm{H}_{2} \mathrm{O}$-forming $\mathrm{NADH}$ oxidase and impact on redox metabolism. Metab Eng 2006, 8:303-314.

7. Berríos-Rivera SJ, San KY, Bennett GN: The effect of carbon sources and lactate dehydrogenase deletion on 1,2-propanediol production in Escherichia coli. J Ind Microbiol Biotechnol 2003, 30:34-40.

8. Ji XJ, Huang H, Zhu JG, Ren LJ, Nie ZK, Du J, Li S: Engineering Klebsiella oxytoca for efficient 2,3-butanediol production through insertional inactivation of acetaldehyde dehydrogenase gene. App/ Microbiol Biotechnol 2010, 85:1751-1758.

9. Zhang YP, Li Y, Du CY, Liu M, Cao ZA: Inactivation of aldehyde dehydrogenase: a key factor for engineering 1,3-propanediol production by Klebsiella pneumoniae. Metab Eng 2006, 8:578-586.

10. Berríos-Rivera SJ, Bennett GN, San KY: Metabolic engineering of Escherichia coli: increase of NADH availability by overexpressing an $\mathrm{NAD}^{+}$-dependent formate dehydrogenase. Metab Eng 2002, 4:217-229.

11. Zhang YP, Huang ZH, Du CY, Li Y, Cao ZA: Introduction of an NADH regeneration system into Klebsiella oxytoca leads to an enhanced oxidative and reductive metabolism of glycerol. Metab Eng 2009, 11:101-106.

12. Berríos-Rivera SJ, San KY, Bennett GN: The effect of NAPRTase overexpression on the total levels of NAD, the NADH/NAD ${ }^{+}$ratio, and the distribution of metabolites in Escherichia coli. Metab Eng 2002, 4:238-247.

13. Liu LM, Li Y, Shi ZP, Du GC, Chen J: Enhancement of pyruvate productivity in Torulopsis glabrata: Increase of $\mathrm{NAD}^{+}$availability. J Biotechnol 2006, 126:173-185.

14. Sánchez AM, Bennett GN, San KY: Effect of different levels of NADH availability on metabolic fluxes of Escherichia coli chemostat cultures in defined medium. J Biotechnol 2005, 117:395-405.

15. Werpy T, Petersen G: Top value added chemicals from biomass, Volume 1: results of screening for potential candidates from sugars and synthesis gas. http://www1.eere.energy.gov/biomass/pdfs/35523.pdf.

16. Xiao ZJ, Xu P: Acetoin metabolism in bacteria. Crit Rev Microbio/ 2007, 33:127-140

17. Xiao Z, Wang X, Huang Y, Huo F, Zhu X, Xi L, Liu JR: Thermophilic fermentation of acetoin and 2,3-butanediol by a novel Geobacillus strain. Biotechnol Biofuels 2012, 5:88

18. Ji XJ, Huang H, Nie ZK, Qu L, Xu Q, Tsao GT: Fuels and chemicals from hemicellulose sugars. Adv Biochem Eng Biotechnol 2012, 128:199-224.

19. Liu YF, Zhang SL, Yong YC, Ji ZX, Ma X, Xu ZH, Chen SW: Efficient production of acetoin by the newly isolated Bacillus licheniformis strain MEL09. Process Biochem 2011, 46:390-394.

20. Xu P, Xiao Z, Du Y, Wei Z: An acetoin high yield Bacillus pumilus strain. European Patent 2009.

21. Zhang X, Yang $T$, Lin Q, Xu M, Xia H, Xu Z, Li H, Rao Z: Isolation and identification of an acetoin high production bacterium that can reverse transform 2,3-butanediol to acetoin at the decline phase of fermentation. World J Microbiol Biotechnol 2011, 12:2785-2790.

22. Ji XJ, Huang H, Ouyang PK: Microbial 2,3-butanediol production: A stateof-the-art review. Biotechnol Adv 2011, 29:351-364.
23. Johansen L, Bryn K, Störmer FC: Physiological and biochemical role of the butanediol pathway in Aerobacter (Enterobacter) aerogenes. J Bacteriol 1975, 123:1124-1130.

24. Afschar AS, Bellgardt KH, Rossell CE, Czok A, Schaller K: The production of 2,3-butanediol by fermentation of high test molasses. Appl Microbiol Biotechnol 1991, 34:582-585.

25. Ji XJ, Huang H, Du J, Zhu JG, Ren LJ, Hu N, Li S: Enhanced 2,3-butanediol production by Klebsiella oxytoca using a two-stage agitation speed control strategy. Bioresour Technol 2009, 100:3410-3414.

26. Nie ZK, Ji XJ, Huang H, Du J, Li ZY, Qu L, Zhang Q, Ouyang PK: An effective and simplified fed-batch strategy for improved 2,3-butanediol production by Klebsiella oxytoca. Appl Biochem Biotechnol 2011, 163:946-953.

27. Qureshi N, Cheryan M: Production of 2,3-butanediol by Klebsiella oxytoca. Appl Microbiol Biotechnol 1989, 30:440-443.

28. Li D, Dai JY, Xiu ZL: A novel strategy for integrated utilization of Jerusalem artichoke stalk and tuber for production of 2,3-butanediol by Klebsiella pneumoniae. Bioresour Technol 2010, 101:8342-8347.

29. Ma CQ, Wang AL, Qin JY, Li LX, Ai XL, Jiang TY, Tang HZ, Xu P: Enhanced 2,3-butanediol production by Klebsiella pneumoniae SDM. Appl Microbiol Biotechnol 2009, 82:49-57.

30. Qin JY, Xiao ZJ, Ma CQ, Xie NZ, Liu PH, Xu P: Production of 2,3-butanediol by Klebsiella pneumoniae using glucose and ammonium phosphate. Chin J Chem Eng 2006, 14:132-136.

31. Sun LH, Wang XD, Dai JY, Xiu ZL: Microbial production of 2,3-butanediol from Jerusalem artichoke tubers by Klebsiella pneumoniae. Appl Microbio Biotechnol 2009, 82:847-852.

32. Wang AL, Wang $Y$, Jiang TY, Li LX, Ma CQ, Xu P: Production of 2,3butanediol from corncob molasses, a waste by-product in xylitol production. Appl Microbiol Biotechnol 2010, 87:965-970.

33. Hou J, Lages NF, Oldiges M, Vemuri GN: Metabolic impact of redox cofactor perturbations in Saccharomyces cerevisiae. Metab Eng 2009, 11:253-261

34. Vemuri G, Eiteman M, McEwen J, Olsson L, Nielsen J: Increasing NADH oxidation reduces overflow metabolism in Saccharomyces cerevisiae. Proc Natl Acad Sci USA 2007, 104:2402-2407.

35. Fournet-Fayard S, Joly B, Forestier C: Transformation of wild type Klebsiella pneumoniae with plasmid DNA by electroporation. J Microbiol Methods 1995, 24:49-54.

36. Ji XJ, Nie ZK, Huang H, Ren LJ, Peng C, Ouyang PK: Elimination of carbon catabolite repression in Klebsiella oxytoca for efficient 2,3-butanediol production from glucose-xylose mixtures. Appl Microbiol Biotechnol 2011, 89:1119-1125.

37. Zhu JG, Li S, Ji XJ, Huang H, Hu N: Enhanced 1,3-propanediol production in recombinant Klebsiella pneumoniae carrying the gene yqhD encoding 1,3-propanediol oxidoreductase isoenzyme. World J Microbiol Biotechnol 2009, 25:1217-1223.

38. Auzat I, Chapuy-Regaud S, Bras GL, Santos DD, Ogunniyi AD, Thomas IL, Garel JR, Paton JC, Trombe MC: The NADH oxidase of Streptococcus pneumoniae: its involvement incompetence and virulence. Mol Microbiol 1999, 34:1018-1028

39. Kleiner D, Paul W, Merrick MJ: Construction of multicopy expreesion vectors for regulated overproduction of proteins in Klebsiella pneumoniae and other enteric bacteria. J Gen Microbiol 1988, 134:1779-1784.

40. Joseph S, David WR: Molecular cloning: a laboratory manual. 3rd edition. New York: Cold Spring Harbor Laboratory Press; 2001.

41. de Felipe FL, Hugenholtz J: Purification and characterisation of the water forming NADH-oxidase from Lactococcus lactis. Intern Dairy J 2001, 11:37-44.

42. Bradford MM: A rapid and sensitive method for the quantitation of microgram quantities of protein utilizing the principle of protein-dye binding. Anal Biochem 1976, 72:248-254.

43. Lilius EM, Multanen VM, Toivonen V: Quantitative extraction and estimation of intracellular nicotinamid dinucleotides in Escherichia coli. Anal Biochem 1979, 99:22-27.

44. Bernowsky C, Swan M: An improved cycling assay for nicotinamide adenine dinucleotide. Anal Biochem 1973, 53:452-458.

doi:10.1186/1754-6834-6-7

Cite this article as: Ji et al:: Cofactor engineering through heterologous expression of an NADH oxidase and its impact on metabolic flux redistribution in Klebsiella pneumoniae. Biotechnology for Biofuels 2013 6:7. 